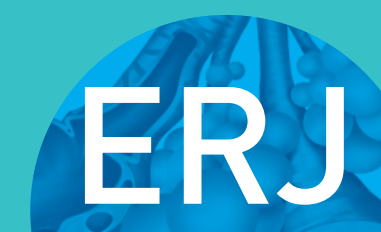

open research

\section{Caspase-1 deficiency reduces eosinophilia and interleukin-33 in an asthma exacerbation model}

\author{
Mandy Menzel ${ }^{1}$, Hamid Akbarshahi ${ }^{1}$, Irma Mahmutovic Persson ${ }^{1}$, \\ Manoj Puthia ${ }^{2}$, Leif Bjermer ${ }^{3}$ and Lena Uller ${ }^{1}$
}

Affiliations: ${ }^{1}$ Respiratory Immunopharmacology, Dept of Experimental Medical Science, Lund University, Lund, Sweden. ${ }^{2}$ Dept of Microbiology, Immunology and Glycobiology, Institute of Laboratory Medicine, Lund University, Lund, Sweden. ${ }^{3}$ Respiratory Medicine and Allergology, Dept of Clinical Sciences Lund, Lund University, Lund, Sweden.

Correspondence: Lena Uller, Dept of Experimental Medical Science, Lund University, BMC D12, 22184 Lund, Sweden. E-mail: Lena.Uller@med.lu.se

ABSTRACT Rhinovirus infections are common triggers of asthma exacerbations. Viruses can activate the inflammasome, resulting in processing and activation of caspase-1. This recruitment triggers production of interleukin (IL)-1 $\beta$ and IL-18, which have been implicated in asthma. Elucidating the involvement of the inflammasome and its compartments, such as caspase-1, in asthma exacerbations is warranted.

Gene expression of caspase-1 was measured in rhinovirus-infected primary bronchial epithelial cells of asthmatic and healthy donors $24 \mathrm{~h}$ post-infection. In an in vivo exacerbation experiment C57BL/6 wildtype and caspase- $1^{-/-}$mice were challenged with house dust mite followed by exposures to the viral mimic poly(I:C). General lung inflammatory parameters and levels of T-helper type 2 (Th2)-upstream cytokines IL-33, thymic stromal lymphopoietin (TSLP) and IL-25 were assessed.

Caspase-1 expression was elevated after rhinoviral infection exclusively in bronchial epithelial cells from asthmatics. In a translational mouse model of asthma exacerbation effects of caspase-1 on airway inflammation and Th2-upstream cytokines were explored. Caspase-1 deficient mice exhibited no alterations of general lung inflammatory parameters, but showed markedly reduced eosinophilia. Furthermore, the Th2-upstream cytokines IL-33, TSLP and IL-25 were reduced at exacerbation in mice lacking caspase- 1 .

Rhinovirus infection increases bronchial epithelial caspase-1 in asthma. Caspase-1 may induce production of lung Th2-upstream cytokines and eosinophilia at exacerbations. Further targeting of caspase-1 signalling is warranted to explore its role in asthma exacerbations.

@ERSpublications

Lack of caspase-1 reduces eosinophilia and expression of Th2-upstream cytokines at experimental asthma exacerbation http://ow.ly/NPMK30eMV2E

Cite this article as: Menzel M, Akbarshahi H, Mahmutovic Persson I, et al. Caspase- 1 deficiency reduces eosinophilia and interleukin-33 in an asthma exacerbation model. ERJ Open Res 2017; 3: 00047-2017 [https://doi.org/10.1183/23120541.00047-2017].

This article has supplementary material available from openres.ersjournals.com

Received: April 072017 | Accepted after revision: Aug 072017

Support statement: This study was supported by financial grants from the Swedish Research Council, the Swedish Heart-Lung Foundation and VINNOVA. Funding information for this article has been deposited with the Crossref Funder Registry.

Conflict of interest: None declared

Copyright $\odot$ ERS 2017. This article is open access and distributed under the terms of the Creative Commons Attribution Non-Commercial Licence 4.0 


\section{Introduction}

Asthma is a chronic respiratory disorder, characterised by wheezing, shortness of breath, chest tightness and airway hyperresponsiveness, affecting $>200$ million people globally [1]. The majority of asthmatics show a T-helper type 2 cell (Th2)-dominant phenotype defined by eosinophilia and atopy; however, phenotypes with non-Th2-like inflammation have been characterised [2]. Eosinophilia correlates with disease severity [3]. During respiratory infection-associated exacerbation eosinophilia can be mixed with neutrophilia or even replaced by it [4].

The epithelium-derived Th2-promoting cytokines interleukin (IL)-33, thymic stromal lymphopoietin (TSLP) and IL-25 play a major role in asthma severity and are elevated in asthmatics [5]. Several genome-wide association studies have highlighted an association between polymorphisms of TSLP, IL-33 and the IL-1 receptor-like 1 locus and asthma [6]. Release of these cytokines can be triggered by allergen challenge and by respiratory viral infections. IL-33, TSLP and IL-25 are drivers of inflammation, and their blockage abrogates inflammation [5].

Stimulation with pathogen-associated molecular patterns (respiratory infections via e.g. Toll-like receptor 3 and RIG-I-like helicases) and damage-associated molecular patterns (e.g. ATP and uric acid) triggers the assembly of a multiprotein complex, the inflammasome. The most studied inflammasome is the NACHT, LRR and PYD domains-containing protein (NLRP)3 inflammasome [7]. Stimulatory triggers result in the recruitment of ASC and pro-caspase-1 to NLRP3, which leads to the processing and activation of caspase-1. Mature caspase- 1 then cleaves the pro-forms of the pro-inflammatory cytokine IL- $1 \beta$ into its active forms, which in turn triggers the release of other pro-inflammatory cytokines [8]. The relevance of IL-1 $\beta$ in asthma has been implicated; asthmatics show higher levels of IL-1 $\beta$ in serum and its role in airway inflammation has been studied in mouse models [7].

We hypothesised that, despite controversial findings, caspase-1 is involved in the production of another IL-1 family member, IL-33, which is involved in Th2-features of asthma exacerbations.

\section{Methods}

Bronchial epithelial cell culture and rhinovirus infection

Cultures of human bronchial epithelial cells (HBECs) from asthmatic donors were obtained by bronchoscopy using a fibre-optic bronchoscope (IT160; Olympus, Tokyo, Japan). The procedure was performed in accordance with standard published guidelines and processed as described previously [9]. Patients provided written informed consent, and approval was obtained from the regional ethical review board at Lund University (Lund, Sweden; permit no. 218/2011). Patients' characteristics are presented in table 1. Additionally, primary bronchial epithelial cells from healthy donors were purchased (Lonza, Walkersville, MD, USA). HBECs were cultured in bronchial epithelial growth medium (Clonetics, San Diego, CA, USA). For experiments, HEBCs were seeded in 12-well plates (Nunc; Life Technologies, Carlsbad, CA, USA) and when $80-90 \%$ confluent, the cell monolayer was infected with 1 multiplicity of infection rhinovirus RV16. Cell lysates were obtained $24 \mathrm{~h}$ post-infection.

\section{Mouse model and study design}

Experiments were approved by the regional laboratory animal ethics committee in Malmö/Lund (permit no. M36-13) and performed as previously described [10]. In short, C57BL/6 wild-type and caspase- $1^{-1-}$ mice (provided by Catharina Svanborg, Lund University, Lund, Sweden) were challenged with $25 \mu \mathrm{g}$ house

\section{TABLE 1 Characteristics of the asthma patients included in this study; all asthmatics were}

atopic

\begin{tabular}{lccc} 
Sex & Age years & FEV $\mathbf{~ \% ~ p r e d ~}$ & Concomitant medication \\
\hline Male & 21 & 89.2 & ICS, SABA \\
Male & 30 & 94.8 & ICS, SABA \\
Male & 39 & 95.7 & ICS, SABA \\
Female & 19 & 115.9 & ICS, SABA \\
Female & 32 & 101.5 & ICS \\
Female & 26 & 102.5 & ICS/SABA \\
Female & 25 & 79.8 & None \\
Female & 25 & 96.3 & None
\end{tabular}

FEV1: forced expiratory volume in $1 \mathrm{~s}$; ICS: inhaled corticosteroids; SABA: short-acting $\beta$-adrenoceptor agonist. 
dust mite (HDM; Greer, Lenoir, NC, USA) 3 days $\cdot w^{-1}$ for 3 weeks and were subsequently exposed to three doses of $50 \mu \mathrm{g}$ polyinosinic:polycytidylic acid (poly(I:C); InvivoGen, San Diego, CA, USA). The experiment was terminated $24 \mathrm{~h}$ after the last poly(I:C) exposure. Lavage of the lungs was performed with PBS and lung tissue was snap-frozen and stored at $-80^{\circ} \mathrm{C}$ until further processing.

\section{Bronchoalveolar lavage fluid analysis}

Cytospin of bronchoalveolar lavage fluid (BALF) was run and stained with May-Grünwald/Giemsa. 400 cells were counted and classified as neutrophils and eosinophils. Total count of BALF cells was performed and total BALF protein concentrations were determined.

Gene expression analysis by quantitative real-time PCR

Total RNA was extracted from HBEC lysates and frozen mouse lung homogenates (Nucleospin RNA II; Macherey-Nagel, Düren, Germany) and was reverse-transcribed to cDNA (Precision nanoScript2 reverse transcription kit; PrimerDesign, Southampton, UK). Then quantitative real-time PCR was run on a Mx3005P qPCR system (Stratagene, La Jolla, CA, USA) with standard cycling parameters. Primers were obtained from PrimerDesign and Qiagen (Sollentuna, Sweden). Samples were analysed using the $\Delta \Delta \mathrm{Ct}$ method [11] and related to UBC/GAPDH expression for in vitro experiments and 18S rRNA for in vivo experiments.

\section{Quantification of Th2-upstream cytokines by ELISA}

IL-33, TSLP and IL-25 levels were measured in mouse lung homogenates according to the manufacturer's instructions (R\&D Systems, Abingdon, UK) and normalised to total protein content of the lung homogenates (BCA assay; Pierce, Thermo Scientific, Waltham, MA, USA).

\section{Immunohistochemistry staining}

Mouse lung tissue was paraffin-embedded and cut into $4-\mu \mathrm{m}$ sections. Sections were blocked with $5 \%$ serum, followed by overnight incubation with a primary goat anti-mouse IL-33 antibody (R\&D Systems) at $4^{\circ} \mathrm{C}$. Then sections were incubated with a secondary donkey anti-goat IgG antibody (R\&D Systems) and staining was visualised with 3,3'-diaminobenzidine (Vector Laboratories, BioNordika, Stockholm, Sweden) and background stained with haematoxylin. Stained sections were scanned (Aperio; Leica Microsystems, Bromma, Sweden).

\section{Statistical analysis}

Data are presented as mean \pm SEM. Statistical analysis was performed in $\mathrm{R}[12]$. p-values $<0.05$ were regarded as statistically significant. Comparison of different groups was performed using the Kruskal-Wallis test, with the Wilcoxon analysis post-testing.

\section{Results}

Caspase-1 expression is induced after rhinovirus infection in primary bronchial epithelial cells of asthmatics

In order to investigate whether caspase- 1 expression is altered after rhinovirus infection, primary bronchial epithelial cells from asthma patients and healthy individuals were infected with the major group rhinovirus RV16. 24 h post-infection gene levels of caspase-1 were assessed. While caspase-1 expression did not differ at baseline between diseased and healthy epithelial cells, there was a significant induction in caspase-1 expression after RV16 infection exclusively in bronchial epithelial cells from asthmatics $(\mathrm{p}<0.01$; figure 1$)$, which was not present in healthy epithelium. Based on our observation we proceeded to study the role of caspase-1 in a mouse model of asthma exacerbation.

\section{Lack of caspase-1 does not affect general lung inflammatory parameters in an experimental} mouse asthma exacerbation model, but reduces eosinophilia while increasing neutrophilia For in vivo studies of the involvement of caspase-1 in asthma exacerbations, wild-type and caspase-1 deficient mice were challenged with HDM, which was followed by subsequent exposure to the viral mimic poly(I:C) (online supplementary figure S1) This model shares features with viral infection-evoked asthma exacerbations, including mixed granulocyte bronchial inflammation [10]. Mice deficient in caspase-1 did not differ in BALF cell count from their wild-type littermates, but had a higher total protein concentration at exacerbation (figure $2 \mathrm{a}$ and $\mathrm{b}$ ). After HDM challenge caspase-1 deficient mice exhibited markedly reduced eosinophilia and neutrophilia $(p<0.05$; figure $2 c$ and $d)$. While lack of eosinophilia was sustained during exacerbation, levels of neutrophils showed a trend to be elevated in mice deficient in caspase-1 (figure $2 \mathrm{c}$ and $\mathrm{d}$ ), changing the inflammatory phenotype. Similarly, gene expression of the eosinophil-recruiting chemokine CCL11 was not increased at exacerbation in knockout mice compared to their wild-type littermates (figure 3). 


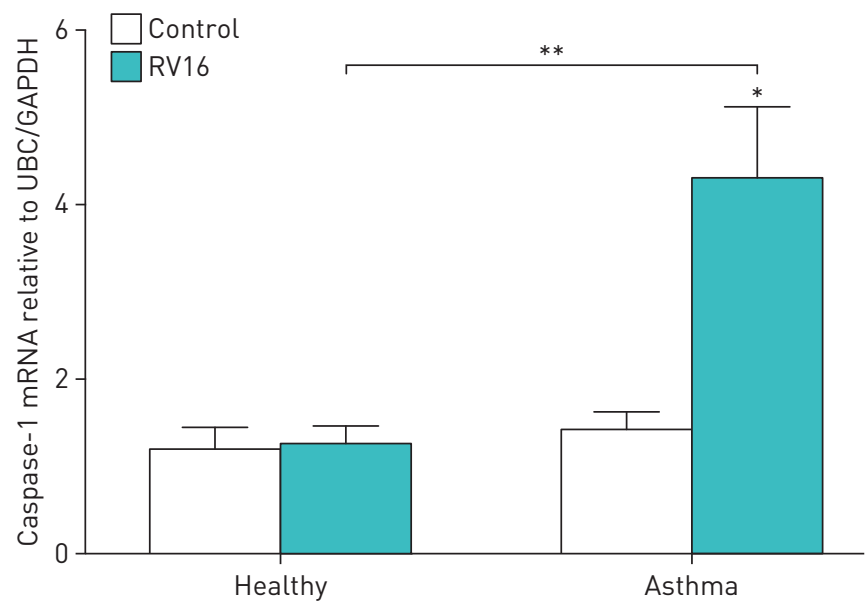

FIGURE 1 Rhinovirus (RV) infection induces caspase-1 expression in bronchial epithelial cells in asthma. Human bronchial epithelial cells from asthmatic and healthy donors were infected with 1 multiplicity of infection RV16 and gene expression of caspase-1 was measured by real-time quantitative PCR $24 \mathrm{~h}$ post-infection. Data are presented as mean \pm SEM fold change of unstimulated healthy control relative to UBC/ GAPDH expression. Comparison of different groups was performed using the Kruskal-Wallis test and Wilcoxon post-testing. ${ }^{*}: p<0.05$ versus healthy subjects; ${ }^{* *}: p<0.01$. Data were obtained from eight asthmatic and four healthy donors.

Lack of caspase-1 reduces protein but not gene levels of IL-33

The IL-1 family member IL-33 is known to be upregulated during asthma exacerbations [5] and is associated with eosinophilia [13]. We thus measured gene and protein levels of this cytokine in mouse lung homogenates. At the gene level IL-33 was upregulated during exacerbations in wild-type and caspase-1 deficient mice. There was a trend towards higher expression of IL-33 in mice lacking caspase-1, although this was not statistically significant (figure 4a). Gene expression of IL-1 $\beta$, another IL-1 family
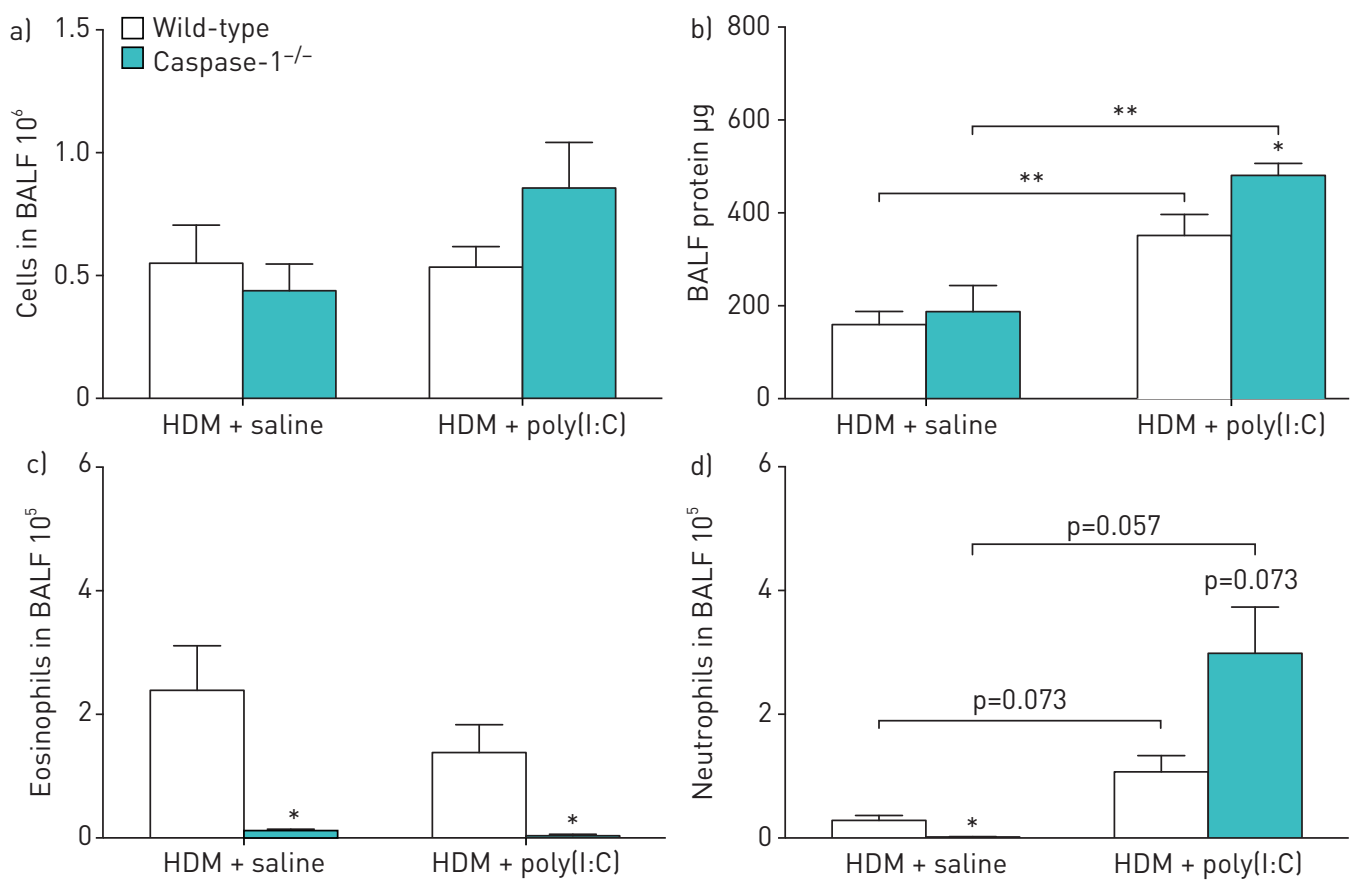

FIGURE 2 Effect of caspase-1 deficiency on lung inflammatory parameters and inflammatory cell subsets in a mouse model of asthma exacerbation. Mice were challenged with house dust mite (HDM) 3 days.week $^{-1}$ for 3 weeks and were then stimulated with polyinosinic:polycytidylic acid (poly(l:C)) or saline for 3 days. Lavage of the lungs was performed using PBS $24 \mathrm{~h}$ after the final poly(l:C) exposure. Bronchoalveolar lavage fluid (BALF) a) cell count and b) protein concentration were measured. Cytospins were stained with May-Grünwald/ Giemsa and cells were counted and classified as c) eosinophils and d) neutrophils. Data are presented as mean \pm SEM. Comparison of different groups was performed using Kruskal-Wallis with Wilcoxon post-testing. ${ }^{*}$ : $p<0.05 ;{ }^{* *}$ : $p<0.01$ versus wild-type. Data were obtained from 5-8 mice per group. 


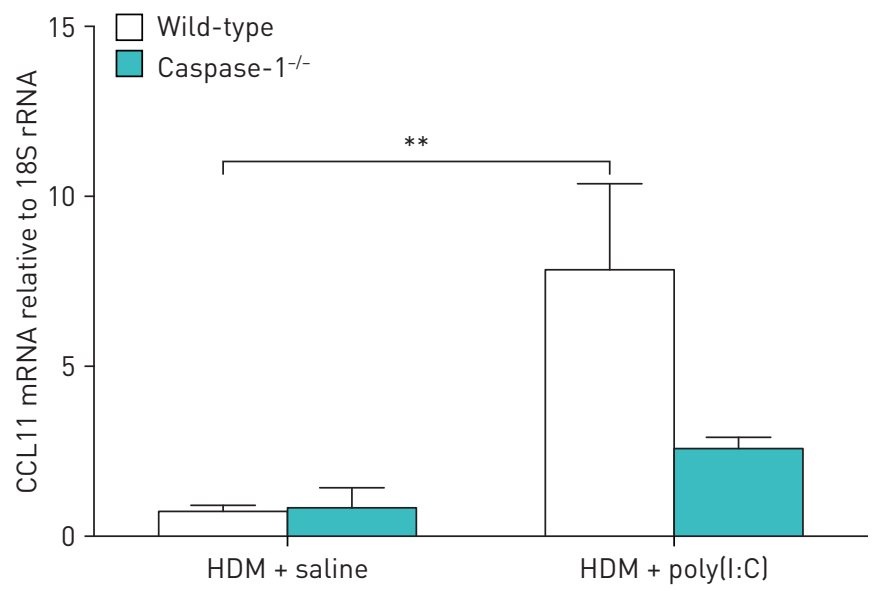

FIGURE 3 Expression of CCL11 is not induced in caspase- 1 deficient mice at exacerbation. Mice were challenged with house dust mite (HDM) 3 days. week $^{-1}$ for 3 weeks and were then stimulated with polyinosinic: polycytidylic acid (poly(I:C)) or saline for 3 days. CCL11 gene expression was measured in lung homogenates. Data are presented as mean \pm SEM. Comparison of different groups was performed using the Kruskal-Wallis test with Wilcoxon post-testing. ${ }^{* *}: p<0.01$. Data were obtained from 5-8 mice per group.

member expressed downstream of caspase-1, was induced at exacerbation in wild-type mice and showed a trend towards higher induction in caspase-1 deficient mice (online supplementary figure S2) and thus followed expression of IL-33. In contrast, protein levels of mature bioactive IL-33 were significantly reduced in exacerbating caspase- 1 deficient mice compared with wild-type mice ( $p<0.001$; figure $4 b)$. In
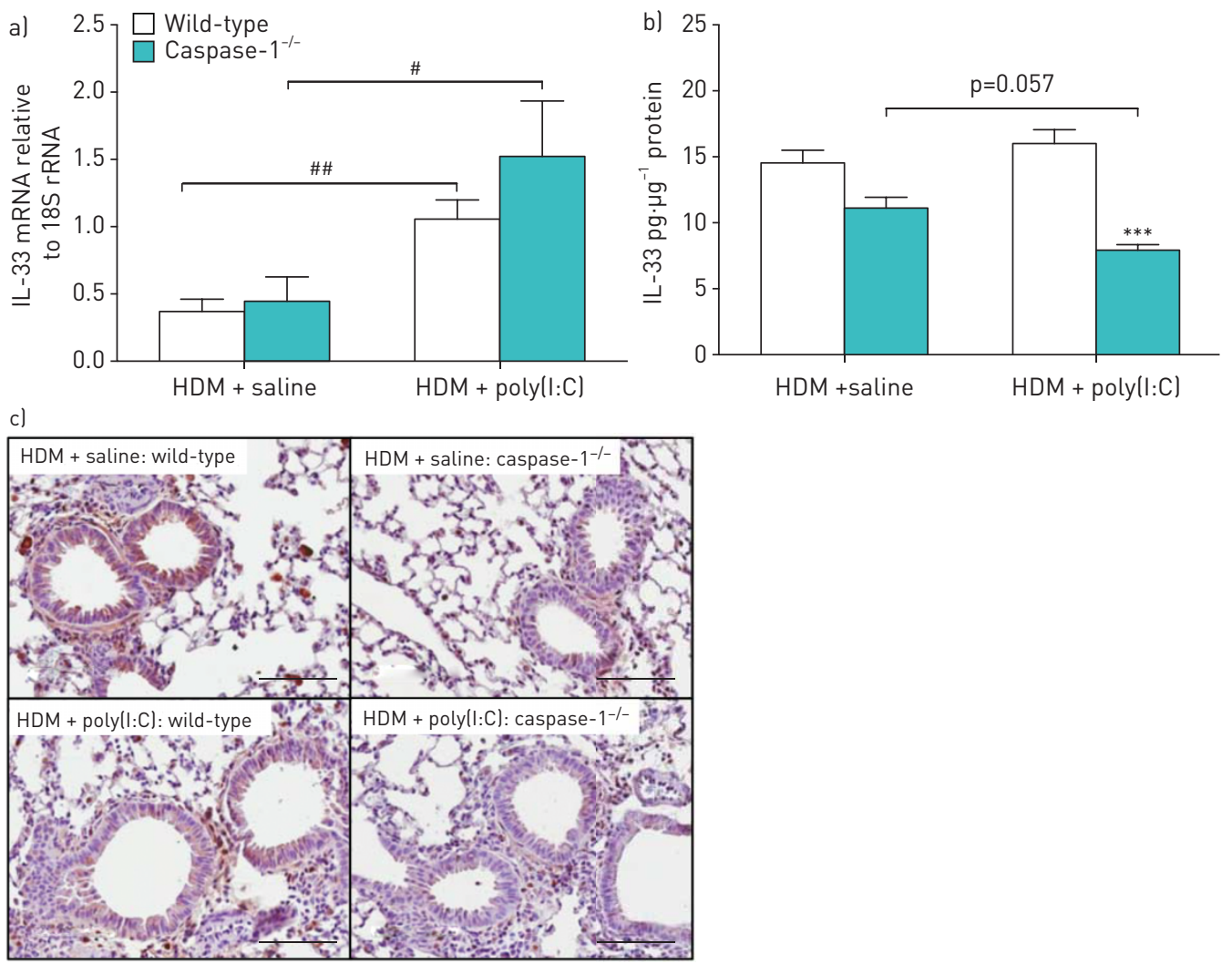

FIGURE 4 Lung interleukin (IL)-33 protein expression in vivo depends on caspase-1. Mice were challenged

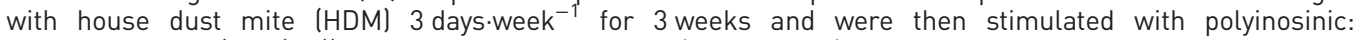
polycytidylic acid (poly(I:C)) or saline for 3 days. IL-33 a) gene and b) protein expression was measured in lung homogenates. Data are presented as mean \pm SEM. Comparison of different groups was performed using the Kruskal-Wallis test with Wilcoxon post-testing. ${ }^{\#}: p<0.05 ;{ }^{\# \#}: p<0.01 ;{ }^{* * *}: p<0.001$ versus wild-type. Data were obtained from 5-8 mice per group. c) A representative image of lung IL-33 immunostaining. Scale bars $=100 \mu \mathrm{m}$. 
addition, reduction of IL-33 expression in mice lacking caspase-1 was detected by immunostaining of mouse lung sections (figure $4 \mathrm{c}$ ).

\section{Lack of caspase-1 reduces expression of the Th2-upstream cytokines TSLP and IL-25}

To further elucidate whether caspase-1 contributes to the expression of other Th2-upstream cytokines, levels of TSLP and IL-25 were measured in lung homogenates. Gene expression of TSLP and IL-25 tended to be induced at exacerbation in wild-type mice, while there was no induction in caspase-1 deficient mice (figure 5a and c). Similarly, at protein level, expression of IL-25 and TSLP was lower at exacerbation in caspase-1 deficient mice compared to their wild-type counterparts (figure $5 \mathrm{~b}$ and $\mathrm{d}$ ).

\section{Discussion}

Activation of the NLRP3 inflammasome emerges to play a main role in airway inflammation and innate immunity in asthma. Viral pathogens, such as rhinovirus, are main triggers of asthma exacerbations and are sensed by the inflammasome [14]. Further studies provide evidence that the NLRP3 inflammsome and caspase- 1 are expressed in the airway epithelium, the first barrier against invading pathogens, and can be activated by allergens [15]. Lack of NLRP3 in a murine allergic asthma model results in reduced airway inflammation and Th2 cytokine production [16]. However, other studies fail to show involvement of NLRP3 on Th2 type inflammation [17, 18]. The role of the NLRP3 inflammasome in asthma remains thus controversial and data on the involvement of NLRP3 in asthma exacerbations are scarce. The present translational data, involving rhinovirus-infected bronchial epithelium from asthmatic donors and a mouse model of viral stimulus-induced exacerbations, support the possibility of a causative role of caspase-1 in Th2 inflammation of asthma exacerbations.

In this study we have demonstrated involvement of the inflammasome in Th2 cytokine expression and immunity in a viral stimulus-induced asthma exacerbation model. Based on our findings that infection with rhinovirus induces caspase- 1 expression exclusively in asthma bronchial epithelium, we employed a translational mouse model of asthma exacerbation involving caspase- 1 deficient mice, and show for the first time that caspase-1 is involved in inducing eosinophilia and CCL11 during exacerbation and is involved in the induced expression of the Th2-upstream cytokines IL-33, TSLP and IL-25. These findings
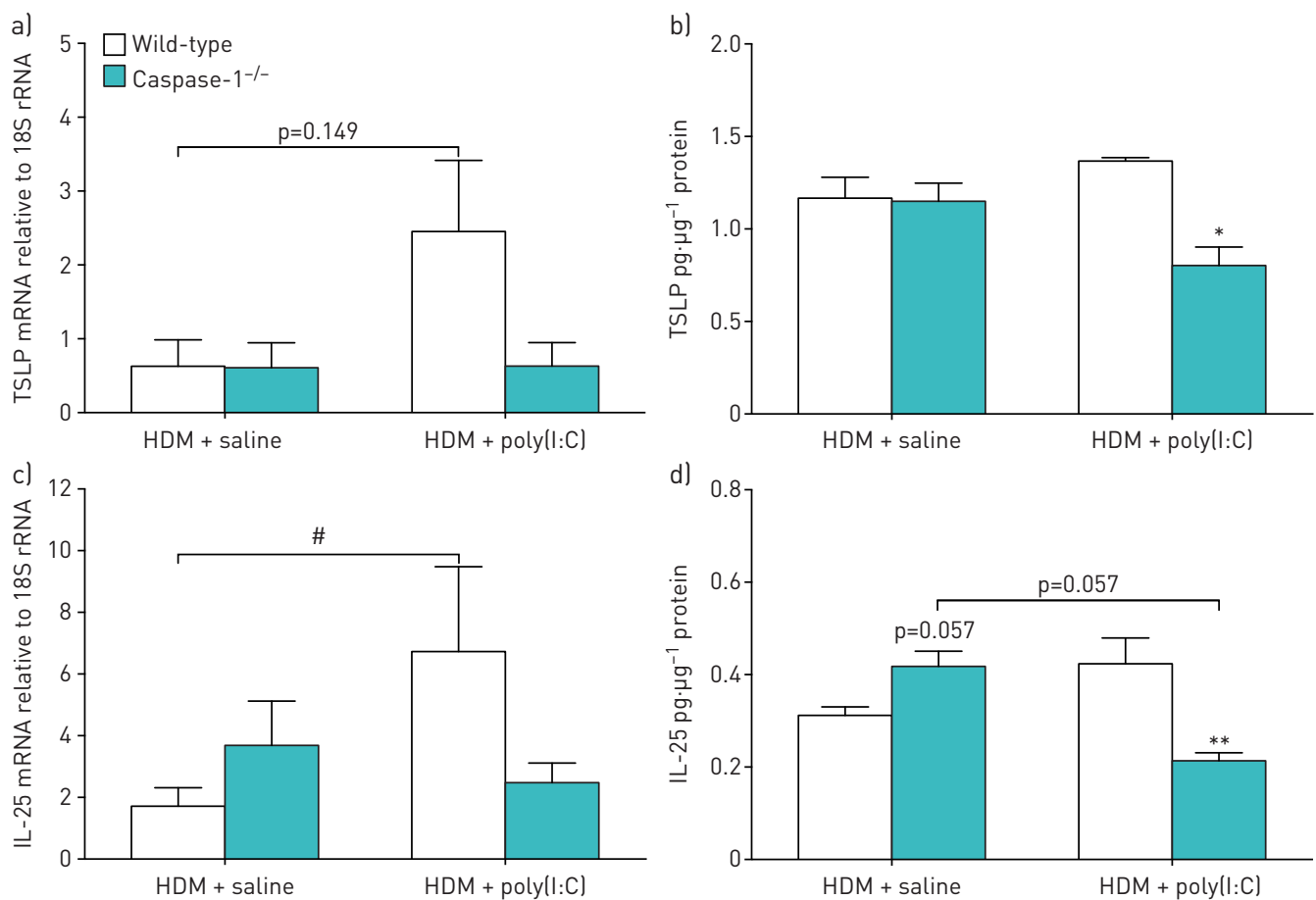

FIGURE 5 Caspase-1 deficient mice have a lower expression of lung T-helper type 2 (Th2)-upstream cytokines at exacerbation. Mice were challenged with house dust mite (HDM) 3 days.week $^{-1}$ for 3 weeks and were then stimulated with polyinosinic:polycytidylic acid (poly(l:C)) or saline for 3 days. Gene $(a, c)$ and protein (b, d) expression, of $a, b)$ thymic stromal lymphopoietin (TSLP) and c, d) interleukin (IL)-25 was measured in lung homogenates. Data are presented as mean \pm SEM. Comparison of different groups was performed using the Kruskal-Wallis test with Wilcoxon post-testing. ${ }^{\#}: \mathrm{p}<0.05 ;^{*}: \mathrm{p}<0.05,{ }^{* *}: \mathrm{p}<0.01$ versus wild-type. Data were obtained from 5-8 mice per group. 
are of interest as treatment options for viral-induced exacerbations are insufficient and a better understanding of the disease and factors involved could contribute to the discovery of novel drug targets.

There is some evidence for the involvement of the inflammasome in the pathogenesis of asthma. Levels of IL-1 $\beta$ are elevated in asthmatics, contributing to airway hyperreactivity [19]. Furthermore, patients with neutrophilic asthma show higher levels of NLRP3 and caspase-1 expression [20]. In addition, infections with respiratory viruses, which are main triggers of asthma exacerbations, can activate the inflammasome. In this study we have shown that experimental infection with rhinovirus in vitro induces caspase-1 expression exclusively in bronchial epithelial cells from asthmatic donors, which is in agreement with a recent report on influenza-induced inflammasome activation in asthma epithelium [21].

Findings from rather pure cell culture experiments can be different from studying whole-lung responses in vivo, where there is interplay of different structural cell types, immune cells and other factors. In order to elucidate the function of caspase- 1 in a whole-lung setting, we employed a translational mouse model of asthma exacerbations [10] involving wild-type and caspase- $1^{-1-}$ mice. Since in our in vitro model rhinovirus infection exclusively induced caspase-1 expression in asthmatic bronchial epithelial cells, we intended to study effects of lack of caspase-1 solely in allergic mice receiving a viral-like stimulus, in order to elucidate a role for caspase- 1 at asthma exacerbation. Furthermore, while the role of the inflammasome or its compartments has been studied in mice with allergic inflammation, to our knowledge there are no studies investigating the role of caspase- 1 in mouse models of asthma exacerbations.

Mice deficient in caspase-1 showed similarities in terms of general inflammatory parameters at exacerbation, measured as cell count and total protein concentration in BALF, compared to their wild-type littermates. However, dissecting the leukocyte levels by differential cell count revealed a different picture: eosinophilia, induced by HDM-challenge in wild-type mice, was completely abrogated in mice lacking caspase-1. At exacerbation, which displayed a mixed neutrophilic-eosinophilic phenotype in wild-type mice, lack of eosinophilia in caspase-1 deficient mice was sustained, while there was a higher influx of neutrophils. Similar to our finding involving the asthma-relevant allergen HDM, in a mouse model of ovalbumin (OVA)-induced allergic inflammation inhibition of the NLRP3 inflammasome [16] or addition of a broad-spectrum caspase inhibitor [22] reduced OVA-induced eosinophilia. Excessive neutrophilia at exacerbation in caspase- $1^{-/-}$mice could be due to a delayed neutrophil apoptosis as suggested by RowE et al. [23].

A high eosinophil count is a predictor of future asthma exacerbations [24]. Eosinophils, in contrast to neutrophils, express the IL-33 receptor ST2 through which IL-33 can activate eosinophils and affect eosinophil survival $[25,26]$. In our model we demonstrate that a reduction in eosinophilia at exacerbation was accompanied with a reduction in bioactive IL-33 in caspase-1 deficient mice. In accordance with our findings, blockage of the NLRP3 inflammasome reduced eosinophilia and IL-33 in a mouse model of OVA-induced allergic inflammation [16]. In contrast, the same group reported that lack of caspase-1 was associated with eosinophilia and increased IL-33 levels in a mouse model of HDM-induced allergic inflammation [27]. While they rightfully point out that unlike OVA challenge, HDM challenge does not require a sensitisation phase, they did sensitise their mice in order to mimic their previous OVA study protocol. It is surprising that a change of allergen would have such opposing effects, despite a similar study protocol. Furthermore, compared to the present study the dosing regimen of HDM in the latter study was shorter and less chronic and included a sensitisation phase of HDM, which could explain differences seen in the inflammatory pattern of both wild-type and caspase- $1^{-1-}$ mice. Technical differences such as the source of HDM extract might as well contribute to the conflicting findings. Post et al. [28] compared the biochemical composition of HDM extract used by MADOURI et al. [27] (ALK-Abello, Hørsholm, Denmark) and in this study (GREER, Lenoir, NC, USA) and found that the ALK-Abello extract had a higher serine/cysteine protease activity than the GREER extract. Furthermore, the GREER extract had a more pronounced effect on epithelial barrier function and induced allergic sensitisation and airway remodelling, suggesting that differences in composition can cause differences in actions of compounds. It should be noted that none of the mentioned studies investigated the effect of caspase- 1 in a model of viral-induced asthma exacerbation.

In contrast to previous studies that investigated the involvement of inflammasome in mouse asthma models using challenges with OVA $[16,22]$, we used repeated challenges with HDM, an allergen relevant to human asthma, to induce airway inflammation without the need for an adjuvant. Challenges with HDM induce mainly eosinophilic inflammation as seen in asthma patients [3]. Infections with rhinovirus have been employed in vivo to model viral-induced exacerbations and have shown success in OVA-challenged mice [29]. However, others have failed to establish an exacerbation phenotype in HDM-challenged mice [30]. In contrast, stimulation with poly(I:C), as in the present study, has emerged as a reproducible method for inducing exacerbation responses in vivo [31]. 
In our model we observed that caspase-1 deficiency did not significantly alter IL-33 gene expression, while levels of mature bioactive IL-33 were reduced. This could be confirmed by staining of paraffin-embedded lung sections. Here, IL-33 expression was reduced in epithelium of caspase-1 deficient mice. However, the involvement of caspase-1 in processing of IL-33 remains controversial [32, 33]. The triad of Th2-upstream cytokines, despite differences in structure and receptor usage, share similar effects on Th2 cytokine expression and can cross-regulate each other [34]. Similar to IL-33, TSLP and IL-25 protein levels were lower at exacerbation in our model in mice lacking caspase-1. Inhibition of caspase-1 has been shown to block TSLP expression in activated mast cells [35].

In conclusion, we show that caspase- 1 expression is exclusively induced in primary bronchial epithelial cells from asthmatic patients by rhinovirus infection. Furthermore, in an experimental mouse model of viral stimulus-induced asthma exacerbation using mice deficient in caspase- 1 we show that caspase- 1 may induce lung protein levels of the Th2-upstream cytokines IL-33, TSLP and IL-25, which is associated with an induction of eosinophilia. It could be speculated that caspase-1 might affect maturity of IL-33, which would potentially lead to changes in eosinophil recruitment. Targeting caspase- 1 signalling is warranted to explore its role in rhinovirus-induced asthma exacerbations. While this study touches on some important aspects of caspase-1 signalling in viral stimulus-induced asthma exacerbations, further studies are warranted, carefully dissecting the exact mechanisms by which this pathway affects Th2 immunity.

\section{Acknowledgements}

Author contributions: M. Menzel, H. Akbarshahi and L. Uller contributed to the conception and design of the work. M. Menzel, H. Akbarshahi, I. Mahmutovic Persson and L. Uller contributed to the acquisition, analysis and interpretation of the work. L. Bjermer provided clinical samples. M. Puthia bred and provided the mice. M. Menzel drafted the manuscript and M. Menzel, H. Akbarshahi, I. Mahmutovic Persson, M. Puthia, L. Bjermer and L. Uller revised the manuscript. All authors have read and approved the submission of the manuscript.

\section{References}

1 World Health Organization. Asthma: Fact Sheet No 307. www.who.int/mediacentre/factsheets/fs307/en/ Date last accessed: December 2016. Date last updated: April 2017.

2 Wenzel SE. Asthma phenotypes: the evolution from clinical to molecular approaches. Nat Med 2012; 18: 716-725.

3 Bousquet J, Chanez P, Lacoste JY, et al. Eosinophilic inflammation in asthma. N Engl J Med 1990; 323: 1033-1039.

4 Fahy JV, Kim KW, Liu J, et al. Prominent neutrophilic inflammation in sputum from subjects with asthma exacerbation. J Allergy Clin Immunol 1995; 95: 843-852.

5 Divekar R, Kita H. Recent advances in epithelium-derived cytokines (IL-33, IL-25, and thymic stromal lymphopoietin) and allergic inflammation. Curr Opin Allergy Clin Immunol 2015; 15: 98-103.

6 Ober C, Yao TC. The genetics of asthma and allergic disease: a 21st century perspective. Immunol Rev 2011; 242: $10-30$.

7 Birrell MA, Eltom S. The role of the NLRP3 inflammasome in the pathogenesis of airway disease. Pharmacol Ther 2011; 130: 364-370.

8 Martinon F, Burns K, Tschopp J. The inflammasome: a molecular platform triggering activation of inflammatory caspases and processing of proIL- $\beta$. Mol Cell 2002; 10: 417-426.

9 Uller L, Leino M, Bedke N, et al. Double-stranded RNA induces disproportionate expression of thymic stromal lymphopoietin versus interferon- $\beta$ in bronchial epithelial cells from donors with asthma. Thorax 2010; 65: 626-632.

10 Mahmutovic Persson I, Akbarshahi H, Menzel M, et al. Increased expression of upstream TH2-cytokines in a mouse model of viral-induced asthma exacerbation. J Transl Med 2016; 14: 52.

11 Livak KJ, Schmittgen TD. Analysis of relative gene expression data using real-time quantitative PCR and the 2- $\Delta \Delta$ CT method. Methods 2001; 25: 402-408.

12 R Development Core Team. R: A Language and Environment for Statistical Computing. Vienna, R Foundation for Statistical Computing, 2013.

13 Stolarski B, Kurowska-Stolarska M, Kewin P, et al. IL-33 exacerbates eosinophil-mediated airway inflammation. J Immunol 2010; 185: 3472-3480.

14 Triantafilou K, Kar S, van Kuppeveld FJ, et al. Rhinovirus-induced calcium flux triggers NLRP3 and NLRC5 activation in bronchial cells. Am J Respir Cell Mol Biol 2013; 49: 923-934.

15 Tran HB, Lewis MD, Tan LW, et al. Immunolocalization of NLRP3 inflammasome in normal murine airway epithelium and changes following induction of ovalbumin-induced airway inflammation. J Allergy 2012; 2012: 819176.

16 Besnard AG, Guillou N, Tschopp J, et al. NLRP3 inflammasome is required in murine asthma in the absence of aluminum adjuvant. Allergy 2011; 66: 1047-1057.

17 Kool M, Willart MA, van Nimwegen M, et al. An unexpected role for uric acid as an inducer of T helper 2 cell immunity to inhaled antigens and inflammatory mediator of allergic asthma. Immunity 2011;34: 527-540.

18 Allen IC, Jania CM, Wilson JE, et al. Analysis of NLRP3 in the development of allergic airway disease in mice. J Immunol 2012; 188: 2884-2893.

19 Zhang Y, Xu CB, Cardell LO. Long-term exposure to IL-1 $\beta$ enhances Toll-IL-1 receptor-mediated inflammatory signaling in murine airway hyperresponsiveness. Eur Cytokine Netw 2009; 20: 148-156.

20 Simpson JL, Phipps S, Baines KJ, et al. Elevated expression of the NLRP3 inflammasome in neutrophilic asthma. Eur Respir J 2014; 43: 1067-1076.

21 Bauer RN, Brighton LE, Mueller L, et al. Influenza enhances caspase-1 in bronchial epithelial cells from asthmatic volunteers and is associated with pathogenesis. J Allergy Clin Immunol 2012; 130: 958-967. 
22 Iwata A, Nishio K, Winn RK, et al. A broad-spectrum caspase inhibitor attenuates allergic airway inflammation in murine asthma model. J Immunol 2003; 170: 3386-3391.

23 Rowe SJ, Allen L, Ridger VC, et al. Caspase-1-deficient mice have delayed neutrophil apoptosis and a prolonged inflammatory response to lipopolysaccharide-induced acute lung injury. J Immunol 2002; 169: 6401-6407.

24 Zeiger RS, Schatz M, Li Q, et al. High blood eosinophil count is a risk factor for future asthma exacerbations in adult persistent asthma. J Allergy Clin Immunol Pract 2014; 2: 741-750.

25 Cherry WB, Yoon J, Bartemes KR, et al. A novel IL-1 family cytokine, IL-33, potently activates human eosinophils. J Allergy Clin Immunol 2008; 121: 1484-1490.

26 Pecaric-Petkovic T, Didichenko SA, Kaempfer S, et al. Human basophils and eosinophils are the direct target leukocytes of the novel IL-1 family member IL-33. Blood 2009; 113: 1526-1534.

27 Madouri F, Guillou N, Fauconnier L, et al. Caspase-1 activation by NLRP3 inflammasome dampens IL-33-dependent house dust mite-induced allergic lung inflammation. J Mol Cell Biol 2015; 7: 351-365.

28 Post S, Nawijn MC, Hackett TL, et al. The composition of house dust mite is critical for mucosal barrier dysfunction and allergic sensitisation. Thorax 2012; 67: 488-495.

29 Bartlett NW, Walton RP, Edwards MR, et al. Mouse models of rhinovirus-induced disease and exacerbation of allergic airway inflammation. Nat Med 2008; 14: 199-204.

30 Rochlitzer S, Hoymann HG, Müller M, et al. No exacerbation but impaired anti-viral mechanisms in a rhinovirus-chronic allergic asthma mouse model. Clin Sci 2014; 126: 55-65.

31 Clarke DL, Davis NH, Majithiya JB, et al. Development of a mouse model mimicking key aspects of a viral asthma exacerbation. Clin Sci 2014; 126: 567-580.

32 Schmitz J, Owyang A, Oldham E, et al. IL-33, an interleukin-1-like cytokine that signals via the IL-1 receptor-related protein ST2 and induces T helper type 2-associated cytokines. Immunity 2005; 23: 479-490.

33 Cayrol C, Girard JP. The IL-1-like cytokine IL-33 is inactivated after maturation by caspase-1. Proc Natl Acad Sci USA 2009; 106: 9021-9026.

34 Saenz SA, Taylor BC, Artis D. Welcome to the neighborhood: epithelial cell-derived cytokines license innate and adaptive immune responses at mucosal sites. Immunol Rev 2008; 226: 172-190.

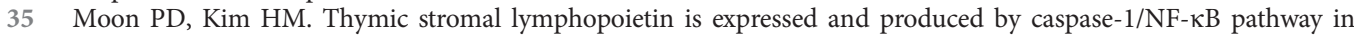
mast cells. Cytokine 2011; 54: 239-243. 